\title{
DES FRONTIÈRES IDÉOLOGIQUES AUX FRONTIĖRES ÉCONOMIQUES: L'Europe et la liberté
}

\author{
Regina Salvador *
}

Presque dix ans après l'effondrement de l'Empire Soviétique on peut bien constater que la fin du conflit idéologique n'était point du tout la fin de l'histoire comme prétendait Fukuyama.

Dans cette période que nous sommes en train de vivre, les conflits culturels n'ont point aussi attendu la profécie catastrophique de Samuel Huntington.

Tout cela nous démonstre la presque incapacité des sciences sociales de prévoir même le futur prochain et les conjunctures de crise. Personne a pu décrive d'avance ce qui se serait passé dans l'Asie du Sud-est et le Japon, en particulier : il nous suffit de regarder les documents d'instutions consagrées telles que la banque Mondiale ou 'Ramses' à titre d'exemple.

On pourrait même dire, pour en citer un philosophe de l'historie que ne jouit pas des faveurs de la pensée hegémonique Hegel que nous sommes en train de constater ce qu'il appellait une "ruse de l'histoire" ("Liste des Vernunft').

Le penseur neo-conservateur Edward Luttwak a dit, en plaisantant, que nous sommes en train de vivre une sorte de révanche de Karl Marx: le facteur économique, determinant en dernière instance, est devenu le facteur unique, déterminant quasi-exclusivement la vie politique et idéologique.

Pendant la période de la Guerre Froide, en quelque sorte, on pourrait isoler les questions politiques des questions économiques; ou, plutôt, les rélations économiques internationales étaient subordinnées à des considérations politiques.

Dans ce contexte, les différends commerciaux ne passaient que d'un sous-ensemble des considérations geostratégiques génèrales, selon les objectifs permanents de la surpuissance en question. C'est à dire, les EUA ont souvent sacrifié ses interêts économiques immédiats dú à des considérations de nature géostratégique.

On peut voir le mème avec la URSS dans ses rapports avec ses États clients.

\section{Le modéle européen}

Est-ce que touts les différends ideologiques et civilisationels puissent être réduits à des questions de rapports de force entre les deux grands acteurs de la Guerre Froide?

Est-ce qu'on peut attribuer exclusivement la construction de l'Europe au Plan Marshall et à la ménace soviétique?

Nous n'en croyons pas.

Nous partageons la conviction que les facteurs politiques et geostratégiques ont étè très importants et, en quelques égards et moments, décisifs mème pour le rédressement des pays de l'Europe Occidentale et du Japon. Néanmoins, le poids des facteurs culturels et civilisationels ont

\footnotetext{
*Professora da Universidade Nova de Lisboa. Departemento de Geografia e Gestảo do Território Tel.: (01) 482.01.15,Fax: (01) 483.59.19,e-maill: regleo@mail.telepac.pt
} 
étè davantage importants, dans la mesure où ils ont étè capables de mobiliser les volontés et les esprits des EUA, qui sont venus au long de ce siècle se battre pour deux fois dans l'Europe pour la défence "d'une certaine idée de la civilisation" dont les interêts économiques n'y sont pour rien.

Cette idée civilisationnelle c'est, à notre avis, l'idée qui a agi comme un moteur dynamique dans l'indépendence et dans la construction de la puissance nord-americaine.

L'idée de la liberté réligieuse, l'auto-gouvernement ("self-government"), l'iluminisme écossais et l'éncyclopedisme français ont étè quelques-uns des piliers de la pensée des Pères-Fondateurs de la sur-puissance Euro-Americaine.

Il faut autant dire que la réalisation des idéaux n'est pas linéaire et non contradictoire: la synthèse n'est toujours pas un processus simple et non douloureux.

L'existence d'un modèle europèen encrée dans l'idée de liberté et de bien-être surpasse des limites de ses frontières géographiques.

C'est vrai que la culture hellénique et la démocratie athénienne constituent des points de repére indéniables. Mais nous savons, depuis le geo-historien Herodote, de l'influence égyptienne dans la réalisation du "miracle grec" Nous savons aussi, grâce à des contributions d'hellenistes français (comme Jean-Pierre Vernant), ou de sociologues (comme Cornelius Castoriadis, d'origine grec), entre autres, que la pensée grecque était bien plus complexe et passible de lectures apologetiques, comme est souvent arrivé avec des humanists allemands ou "oxfordians" (Sir Ernest Barker ou Werner Jaeger).

Par ailleurs, c'est le remarcable philosophe allemand de droit, Hans Kelsen, qui a bien décrit la pluralité des positions politiques, philosophiques et morales des philosophes grecs, comme par exemple, dans la question de la femme (où Xenofonte s'oppose à la mysogenie de Platon et Aristote).

La diversité de conceptions des auteurs grecs peut bien servir de preuve du sens de la liberté, fondement de la civilisation européenne.
Le droit romain a étè la super-structure nécessaire à l'essor du capitalisme moderne et de l'avance économique et technologique qui a permis la réalisation du mythe promethèen.

D'autre part, il faut bien insister que le modèle européen ne doit pas qu'à Athénes et à Rome son existence, en tant que paradigme d'une civilisation.

La contribution du personnalisme cheréten füt une autre source idéniable sans laquelle l'État de Droit ne pourrait pas exister. Le Moyen Âge cherétien occidental avec ses cathédarales, ses monastères et, sourtout, ses Universités s'est developpé simultanement avec des villes "libres" déssinant une poliarchie où l'éparpillement des pouvoirs a forgé les conditions matérielles pour une civilisation conflitueuse et plurielle.

Le rôle de l'Église Catholique et de plusieurs écoles de pensée, comme le nominalisme, la "théologie de la liberté" de Duns Scott, ou même la pensée thomiste, sans parler des percurseurs de l'Humanisme renascentiste comme le Cardinal Nicolas Cusanos, ont étè de vraies piliers de la pensée occidentale moderne.

\section{L'individualisme économique}

L'avènement de la bourgeoiosie et la création d'une civilisation matérielle capitaliste fúrent aussi les conditions nécessaires pour l'épanuissemest du concept de liberté.

Sans une forte classe moyenne et une réprodution élargie de la richesse, les valeurs qui vont constituer l'idéal européen ne pourraient jamais se materialiser. La Réforme protestante a approfundi l'individualisme anti-bureaucratique que, comme nous savons grâce à la thèse de Max Weber, a contribué dans la criation et développement de la societé civile, échellon intermediaire entre le pouvoir publique et la famille.

La societé civile est devenue la condition de possibilité de participation et décision du sujet devenu citoyen, comme était prévu dans l'idéal democratique athénien, connu sous le nom de "boulè" 
Le développement d'une économie-monde a élargi cet individualisme, en universalisant ces idéaux, malgré les visibles contradictions, fruit de la surexploitation des peuples noneuropéens. Malgré tout cela, seront ces mêmes idéaux de liberté qui vont permettre aux victimes de l'économie du monde occidentale se battre pour réaliser idéaux prechés par leurs maitres.

\section{La géographie, ça sert, d'abord à faire le commerce}

Le processus de croissance européenne a étè fait par la violence, comme on a souligné ci-dessus.

Néanmoins, dans ce qui concerne la science de l'espace, elle n'a pas debutée comme la science de États-Majeurs. Dans l'ancienne Babylonie ou dans l'Égypte, les géographes étaient valorisés par sa capacité de prévoir les floues des rivages et les changements climatiques, ainsi que par son activité d'arpenteurs.

Dans la Grèce, à sa foi, géographe était synonime de cosmographe. Il était une sorte de savant universel, spécialist simultanéament dans les siences naturelles et sociales. Ils étaient aussi des cartographes qui répondaient aux besoins du commerce des villes marchandes grecques.

Seul plus tard, avec Alexandre et l'Empire Romain, les déssinateurs de mappes sont devenus serviteurs des génèraux.

Les géographes arabes, comme Ibn Battuta (1304/1356) étaient, avant tout, grands voyageurs et possédeurs d'un esprit encyclopédique qui s'intéressait, avant tout par la connaissance de peuples (comme Battuta remarque dans ses livres de mémoires).

$\mathrm{Au}$ siècle $\mathrm{X}$, soit trois siècles avant Marco Polo (1254/1324), le marchand arabe Souleiman donne une première description de la Chine.

A la cour de Roger II de Sicile, le grand géographe arabe Idriss composait le "Livre de Roger" qui, avec ses cartes, a constitué la discription la plus elaborée du monde médievale.
Cette cartographie s'est basée sur une détermination mathématique de latitude et longitude, qui marchait en parallèle avec une atmosphére de ferveur religieuse. Les géographes arabes aimaient rappeler le propos d'un compagnon aimé du prophéte, Ibn-Saïda : "Le sermon le plus éloquent est encore de voyager à traves le monde sauvage et contempler le repos du marais"

La grande majorité des géographes arabes, qui ont étè fondamentales pour la découverte du chemin maritime des Indes, $n$ 'a jamais étè au service de l'expansion militaire islamique et, beaucoup entre eux, ont méme servi les interêts des marchands chrétiens.

D'autre fois, il faut reconnaitre que le développement de l'art militaire et de la pensée géostrategique ont beaucoup fait pour l'essor de la cartographie. De toute façon, comme disait Clausewitz dans son Livre III (Troisième Chapitre) de son chef-d'oeuvre "De La Guerre" on peut définir celle-ci comme un acte de la vie sociale, "comme une espèce de commerce, a une grande échelle"

Le concept occidental de liberté s'éloigne de l'acte pure de la conscience.

Elle sera toujours une transaction, une échange entre le contigent et la necessité.

Méme le philosophe de la conscience et de la liberté, Jean-Paul Sartre, a dú admettre les limitations de la contigence.

La liberté sera aussi la conscience de la necessité.

\section{La construcion européenne}

L'Historie du développement des sciences et des tecnologies nous a fait apprendre que l'espace pluriel, la tolérance, c'est à dire, le concept de liberté n'est pas notion vaseuse ou métaphysique.

On a vu, par exemple, le retard du développement scientifique soviétique dû à la nature non-libérale du régime. On connait les obstacles au développement de la génétique et de la cyber- 
nétique, à cause des anathèmes proferés par l'Ácadémie de sciences de l'URSS sur le caractère "non dialectique" de ces disciplines accusées d'ètre "neo-kantiennes et idéalistes. Cela va sans dire dans ce concerne les sciences sociales.

Le développement scientifique, donc économique, exige un espace pluriel. La capacité d'administer les conflits a été, jusqu'ici, un presque-monopole de la civilisation Euro-Americaine.

A la fin de la Ilème.Guerrre Mondiale, quand l'Europe a fini sa deuxième guerre civile dans un délai de trente ans à peine, plusieurs representants de l'intelligentzia et des hommes politiques se sont mis d'acccord pour bâtir le rêve saint-simonien d'une Féderation Européenne.

Il faut, tout d'abord, reconnaitre le rôle des disciples de D.Luigi Sturzo, le fondateur de la démocratie-chrétienne italienne et l'inspirateur du rhénan Konrad Adenauer.

La question sociale n'était plus un previlège des forces de gauche. Le social est devenu, comme dans une opération de syntèse, une partie intégrante du concept de liberté. L'économie sociale de marché, défendue par Adenauer et souscrite par Robert Shuman, Jean Monnet et Alcide di Gasperi, répondait au défi du mouvement ouvrier, en l'intégrant aux décisions des societés industrielles, comme propugnaient libérales (comme Stuart Mill) et conservateurs-sociales (comme Auguste Comte).

Le modèle du capitalisme rhénan a étè adopté mème dans le pays du capitalisme "manchesterlen" : le "Welfare State" de Lord Beverage consagralt, d'une certaine façon, les modèles mentionnés ci-dessus.

Aussi, il faut pas oublier le rôle des forces laiques et libérales, representées par le Président de l'Italie Luigi Euinada et par le libéral de gauche Altieri Spinelli. Mais, plus que quelqu'un, c'ètalt le conservateur et franc-maçon Winston Churchill qui, par le poids de son rôle historique, a declanché un mouvement irreversible pour la création de l'Union Européenne.

En somme, toutes les fractions qui répresentent la pensée européenne, comprise comme un idéal de tolerance et d'acceptation de l'altérité, y étaient.

\section{Le possibilisme, paradigme de la science contemporaine}

À guise de conclusion, il nous faut rapeller l'importance de la pensée scientifique qui nous avons mentionné comme un des piliers de l'idéal européen.

On constate, aujourd'hui, que la "cité savante" - soit des humanités, soit de la nature-s'est mis d'accord pour se débarasser du fatalisme ou des logiques déterministes qui dominaient la pensée scientifique au siècle auparavant.

L'indéterminisme de Heisenberg à l'École de Copenhague, la logique du cercle de Vienne, la cybernétique de Betand Lafy ont consagré les paradigmes de la probalité et la causalité stochastique.

Or, il faut pas olublier ce qui arrive souvent la dette de gratitude à la géographie francaise qui a introduit, dans la dialectique des faits humains et de la nature, une vision non-déterministe.

Cette vision, du point de vue épistemologique, n'était peut-ètre pas explicitement elaborée. Cependant, sa contribution a étè plus que rélevante dans la production des connaissances sur l'habitat de l'homme.

Géographes d'appartenances politiques très différentes, comme le communard Elisée Reclus ou le conservateur La Blache ont réflichi d'une façon similaire et dans la mème perspective mème si ce n'était pas explicite que le géographe et philosophe prussien Immanuel Kant, père de la pensée scientifique contemporaine. 


\section{Bibliografia}

ART, Thimothy. "The Threat to Europe" in: Foreign Affairs, March, 1998.

CARTOU, Louis. Communautés Euro-péennes. Paris, Dalloz, 1986.

CLAVAL, Paul.). Géographie Humaine et Économique Comtemporaine. Paris, PUF Fondamental, 1984.

Éleménts de Géographie Humaine. Paris, Éditions M.Th.Génin, 1974.

Principes de Géographie Sociale. Paris,
Éditions M.Th.Génin, 1973.

HOLT, P.M. / LAMBTON, K.S./ LEWIS, B. (Ed.) The Cambridige History of Islam. vol.2 B, Cambridge University Press.

LACOSTE, Yves. La Géographie, Ça Sert, D’Abord à Faire la Guerre. Paris, Editions La Découverte, 1985.

SILLS, David L. (Ed.). International Encyclopedia of the Social Sciences. Vol.5. New York, 1968. 